\title{
Prosopographie et biographie : regards croisés sur la magistrature coloniale belge
}

Enika Ngongo, Bérengère Piret, Laurence Montel \& Pascaline le Polain de Waroux

Enika Ngongo : Enika Ngongo est diplômée en Histoire contemporaine à l'Université catholique de Louvain (2012). Après avoir occupé le poste d'assistante de recherches au sein du Centre d'histoire du droit et de la justice (CHDJ-UCL), elle réalise une thèse de doctorat centrée sur le Congo belge durant la Première Guerre mondiale au sein du Centre de Recherches en Histoire du Droit et des Institutions (CRHiDI) de l'Université Saint-Louis - Bruxelles. Ses intérêts se portent également sur le phénomène de délinquance juvénile au Congo belge et l'enseignement de l'histoire coloniale en Belgique.

Bérengère Piret : Bérengère Piret a consacré sa thèse de doctorat en histoire à l'organisation judiciaire de l'État indépendant du Congo et du Congo belge d'une part et d'autre part à leur activité répressive à partir du cas d'étude qu'est le tribunal de district de Stanleyville (aujourd'hui Kisangani). Elle est par ailleurs l'auteure de plusieurs publications consacrées au droit et aux institutions pénales coloniales, dont l'ouvrage Les cent mille briques. La prison et les détenus de Stanleyville.

Laurence Montel : Laurence Montel est maîtresse de conférences en histoire contemporaine à l'Université de Caen. Ell est également chercheuse associée au centre TELEMME (UMR 7303, AixMarseille Université), de l'ORDCS (Observatoire régional de la délinquance et des contextes sociaux en région PACA), du GIS d'histoire maritime. Ses axes de recherches portent sur les villes portuaires (19e-20e siècles), la police et la justice (19e siècle) et le monde criminel.

Pascaline le Polain de Waroux : Diplômée en histoire contemporaine à l'Université catholique de Louvain (2010), Pascaline le Polain s'est intéressée à l'histoire de la magistrature coloniale belge dans le cadre du projet «Belgian Magistrates (Réseaux sociaux des magistrats belgafricains) ». Au cours de ses précédentes recherches, elle s'est également intéressée au développement de l'Histoire médicale en Afrique Sub-Saharienne, l'Histoire du Sida en Afrique du Sud et à la répression du travail forcé au Congo belge. Elle est actuellement gestionnaire de projets en développement durable à la commune de Woluwe Saint Pierre.

DOI: $10.25518 / 1370-2262.356$

\section{Résumé :}

L'article présente l'avancement et les perspectives du projet « Belgafrima : prosopographie des magistrats coloniaux » (Belgafrican Magistrates) qui élargit au terrain ultramarin la prosopographie des magistrats belges et l'établissement de la carte judiciaire. Le but étant d'entreprendre, à partir des données biographiques saisies, une étude du corps judiciaire colonial. Celle-ci doit mettre en évidence les carrières et réseaux de ces membres tout en prêtant attention aux contraintes collectives comme aux stratégies individuelles. Il est d'abord fait retour sur les sources de cette recherche et sur les difficultés techniques que cette extension du sujet a posées à une base de données pensée à l'origine pour la magistrature métropolitaine. 
Prosopographie et biographie : regards croisés sur la magistrature coloniale ...

En contrepoint de cette analyse prosopographique, le présent article s'attache également à mettre en exergue le parcours d'Antoine Sohier, membre éminent de la magistrature coloniale.

Mots-clés : Antoine Sohier, Biographie, Congo belge, État indépendant du

Congo, Juridictions, Magistrature, Prosopographie

\begin{abstract}
:
Since the start of the twenty-first century, the study of « colonialism » in Belgian Congo has experienced new impulses. Nowadays, the study of judicial body and its staff is also one of the fields of study that is gaining importance. However, this research is not as rich as that relating to the former British and French empires. In 2011, the «Belgafrican Magistrates » project was set up to fill these gaps. The main objective was to initiate, from the biographical data entered, a study of colonial justice. This should highlight the careers and networks of these members while paying attention to the collective constraints on individual strategies. This article develops the progress and prospects of this project. The first part made back to the sources of this research and the methodological and technical issues that are still to be solved. In counterpoint to this prosopographic analysis, the second part of this article highlights the career of Antoine Sohier, eminent member of the Belgian colonial magistracy.
\end{abstract}

Keywords : Antoine Sohier, Biography, Congo, Congo Free State, Judicial body, Jurisdictions, Prosopography

\title{
Introduction 1
}

Depuis une quinzaine d'années, l'étude du «fait colonial » en Belgique connait un nouvel élan $\underline{2}$. Certains thèmes, incontournables pour la période léopoldienne, sont aujourd'hui bien connus (Conférence de Berlin de 1885, « Scramble for Africa », « Red rubber » et « Rapport Casement »). D’autres aspects de cette histoire sont également très travaillés comme par exemple les missions religieuses, l'enseignement, l'économie, les mouvements sociaux, ou encore l'histoire militaire. Les appareils judiciaires et leur personnel comptent aujourd'hui également parmi les chantiers en expansion $\underline{3}$. Toutefois, les recherches en la matière sont moins riches que celles relatives aux anciens empires britannique $\underline{4}$ et français $\underline{5}$. Or, cette question est centrale dans l'histoire de la colonisation belge, trop souvent associée, sinon réduite, à un modèle léopoldien dont le stéréotype s'éloigne d'une quelconque conception de la justice $\underline{6}$. Pourtant, alors que le droit et son application assurent pour l'État colonisateur le contrôle des territoires conquis et de leurs populations en permettant le maintien de sa domination, les magistrats sont, avec les polices et les armées, mais avec des nuances qui restent à établir, des agents de cette domination dont l'étude restait encore en friche. Quelles motivations les poussent à intégrer l'administration colonialez ? Comment se déroulent leurs carrières ? Assiste-t-on à la formation progressive d'un groupe socioprofessionnel homogène par sa formation et son expérience ? Enfin, ce territoire de l'histoire parait aujourd'hui d'autant plus essentiel qu'il offre la possibilité de réinvestir sous d'autres angles l'histoire des sociétés impériales $\underline{8}$ et des États métropolitains, à un moment où l'on interroge dans les deux sens les transferts de modèles, de techniques et de pratiques, entre les métropoles et les colonies.

Dans cette perspective scientifique, le projet «Prosopographie des magistrats belges 1795-1960»(Belgian Magistrates) s'est ouvert aux espaces ultramarins dans le cadre du projet «Belgafrima : prosopographie des magistrats coloniaux belges » (Belgafrican Magistrates). 
Entre 2011 et 2013, la priorité a été donnée à l'étude de l'installation de cette justice et de ses premiers magistrats pendant la période de l'État indépendant du Congo (1885-1908) . Longtemps restés dans l'ombre, les acteurs du droit sont donc au centre de cette recherche. L'exploitation de sources inédites et le recours à une base de données relationnelle inégalée en Belgique représentent en effet une porte d'entrée majeure pour une meilleure compréhension du corps judiciaire, de sa construction et des enjeux de carrière qui s'y jouent.

Si les hommes sont au cœur de la recherche, il s'agit aussi de progresser dans la connaissance de l'organisation et de la carte judiciaire ultramarine qui peinent encore à être vues dans leur ensemble. Intégrer ces informations dans une base de données vise, dans un premier temps, à renouer avec la connaissance de la justice coloniale dont nous avions perdu toute trace jusqu'il y a peu. Elle ambitionne également de faciliter l'étude comparative et relationnelle des justices métropolitaine et ultramarine, que ce soit sur le plan de leur personnel, comme de leurs modèles. Dans quelle mesure les deux corps de magistrats sont-ils, en effet, disjoints, ou ouverts l'un à l'autre ? Quelles furent les influences réciproques des deux systèmes judiciaires ? On sait par exemple que le modèle colonial africain s'inspire tout au long de son existence du modèle métropolitain (importance du parquet, compétences de juridictions et de fonctions)10. Inversement, les transformations de la justice métropolitaine ont pu s'inspirer des innovations africaines, notamment lors des périodes troublées de guerre et d'après-guerre quand les principes mis en œuvre en Afrique ont été appliqués en métropole11. Ensuite, l'inscription de cette recherche coloniale dans l'ère numérique grâce à un recours systématique à une base de données vise à lui donner une visibilité inédite au niveau international. De l'outil proposé et les méthodes de traitement comme d'analyse afférentes nait une histoire coloniale « connectée », ponctuée d'expériences et de regards croisés. Ce vaste projet de recherche et de documentation contribue dès lors à éclairer un champ d'études en pleine construction.

Cet article exploratoire a donc pour objectif de présenter les premiers résultats du projet Belgafrican Magistrates pour l'étude du corps judiciaire colonial belge. Il propose demettre en lumière la magistrature coloniale grâce à deux approches complémentaires : la biographie et la prosopographie. Conjuguées, elles mettent en évidence les carrières et réseaux de ces membres tout en prêtant attention aux contraintes collectives comme aux stratégies individuelles. Le parcours singulier du magistrat Antoine Sohier (1885-1963), partagé entre les continents africain et européen, donne à voir sans pareil toutes les potentialités de l'étude des interconnexions et des ancrages offertes par la base de données. Sa carrière met en effet en évidence la présence de réseaux d'influence importants. De plus, en étant actif au Congo de 1908 à 1934, Sohier s'inscrit dans la continuité directe des magistrats de l'État indépendant du Congo (ÉIC) puisque, bien qu'étant alors formellement une colonie belge, le régime léopoldien se poursuit jusque dans les années 1920. Malgré le changement de régime, les structures et les hommes institués par Léopold II restent en place.

\section{Des sources inédites}

Pour nourrir l'histoire de la magistrature coloniale, les premières sources mobilisées ont été les sources imprimées telles que les Bulletins officiels de l'État indépendant du Congo, les Bulletins officiels du Congo,les Bulletins officiels du Ruanda-Urundi, l'Annuaire du Congo belge etle Recueil mensuel de l'État indépendant du Congo $\underline{12}$, publiés par le gouvernement belge. Les recueils de législation, de jurisprudence et de doctrine, généralement écrits par les magistrats 
Prosopographie et biographie : regards croisés sur la magistrature coloniale ...

coloniaux eux-mêmes, ont également été au centre du dépouillement13. En effet, à l'heure actuelle, l'accès aux sources primaires est encore particulièrement difficile. D'une part parce que les dossiers des agents de l'État indépendant du Congo sont introuvables, probablement détruits (en partie) par l'administration coloniale à la mort de l'agent, ou à la fin de l'ÉIC14. Et d'autre part, parce que les quelque 400 dossiers personnels des magistrats ayant officié dans les colonies et protectorats belges après 1908 ne sont toujours pas libres de consultation du fait de certaines informations « sensibles » relatives à l'agent qu'ils pourraient contenir telles que : ses évaluations par ses supérieurs hiérarchiques, ses éventuelles sanctions, ses liens relationnels, ses préférences politiques. Aussi, si les informations de type strictement identitaire peuvent être encodées et rendues partiellement publiques, il n'en est pas de même pour ces données, du moins pas sans l'autorisation de la Commission de la protection de la vie privée. Certes, ces dernières peuvent faire l'objet d'une recherche scientifique, si elles sont rendues anonymes, mais il parait contestable de les rendre accessibles à tout internaute, sans restriction d'accès, dans la mesure où ces données pourraient être instrumentalisées à des fins étrangères à toute recherche, voire polémiques ou malveillantes. La question touche à la problématique du droit à l'oubli15. Elle s'inscrit en particulier dans le caractère toujours actuel et passionné de l'histoire coloniale en Belgique. Or l'intention qui préside à la réalisation de cette base de données au service de la communauté des chercheurs doit la préserver de ce type de détournement. C'est pourquoi la mise en œuvre d'un système technique et d'un protocole de contrôle modulable d'accès aux données s'est imposée.

En raison de ces contraintes, il fut décidé de travailler dans un premier temps à partir des sources imprimées et des sources libres de droit. Parmi les premières, on trouve un grand nombre de textes relatifs à l'organisation et au personnel judiciaires : création ou mutation de juridiction, délimitation de ressorts, nomination d'agents, etc. Les magistrats actifs au Congo durant les années 1920 peuvent par exemple être appréhendés dans l'Annuaire du Congo belge tandis que les juristes partis pour le Congo belge dans la période comprise entre 1930 et 1960 sont mentionnés dans le Bulletin officiel du Congo belge. Si grâce à ces sources, on peut identifier les principales réformes et les textes règlementaires qui encadrent le travail de la justice, elles présentent toutefois un certain nombre de limites. Les périodes de guerre ne sont souvent pas documentées, de même que les périodes transitoires comme le sont les années 1908 - '1920. Le Bulletin officiel du Ruanda-Urundi n'est par exemple disponible qu'entre 1924 et 1962. Par ailleurs, les décrets et les arrêtés, en matière d'organisation judiciaire ou de sujets connexes, n'y sont pas systématiquement consignés. Le fait est certain pour la période de l'ÉIC et les premières années de la colonie belge que nous traiterons dans le cadre de cet article. Deux volumes de Décrets de l'État indépendant du Congo non publiés au Bulletin officiel édités ensuite en témoignent16. C'est parmi ces textes que se trouve un Règlement général du personnel d'État en Afrique, manifestement paru en 1888, et utile pour connaitre les premières règles établies dans le domaine des carrières coloniales 17 . Ces collections d'arrêtés et de décrets complémentaires ne comblent toutefois pas la totalité des manques qu'un dépouillement systématique des Bulletins officiels de l'ÉIC fait constater. Et, il est vain d'espérer reconstituer, à partir de ces sources, l'ensemble des carrières des magistrats coloniaux, avec leurs bifurcations et leurs cumuls multiples. Néanmoins, les recueils mensuels publiés, dès 1895, par le gouvernement de Boma, les complètent heureusement, de même que les sources libres d'accès - bien que ces dernières soient souvent lacunaires et que certaines ne fournissent des indications que pour certaines périodes $\underline{18}$. 


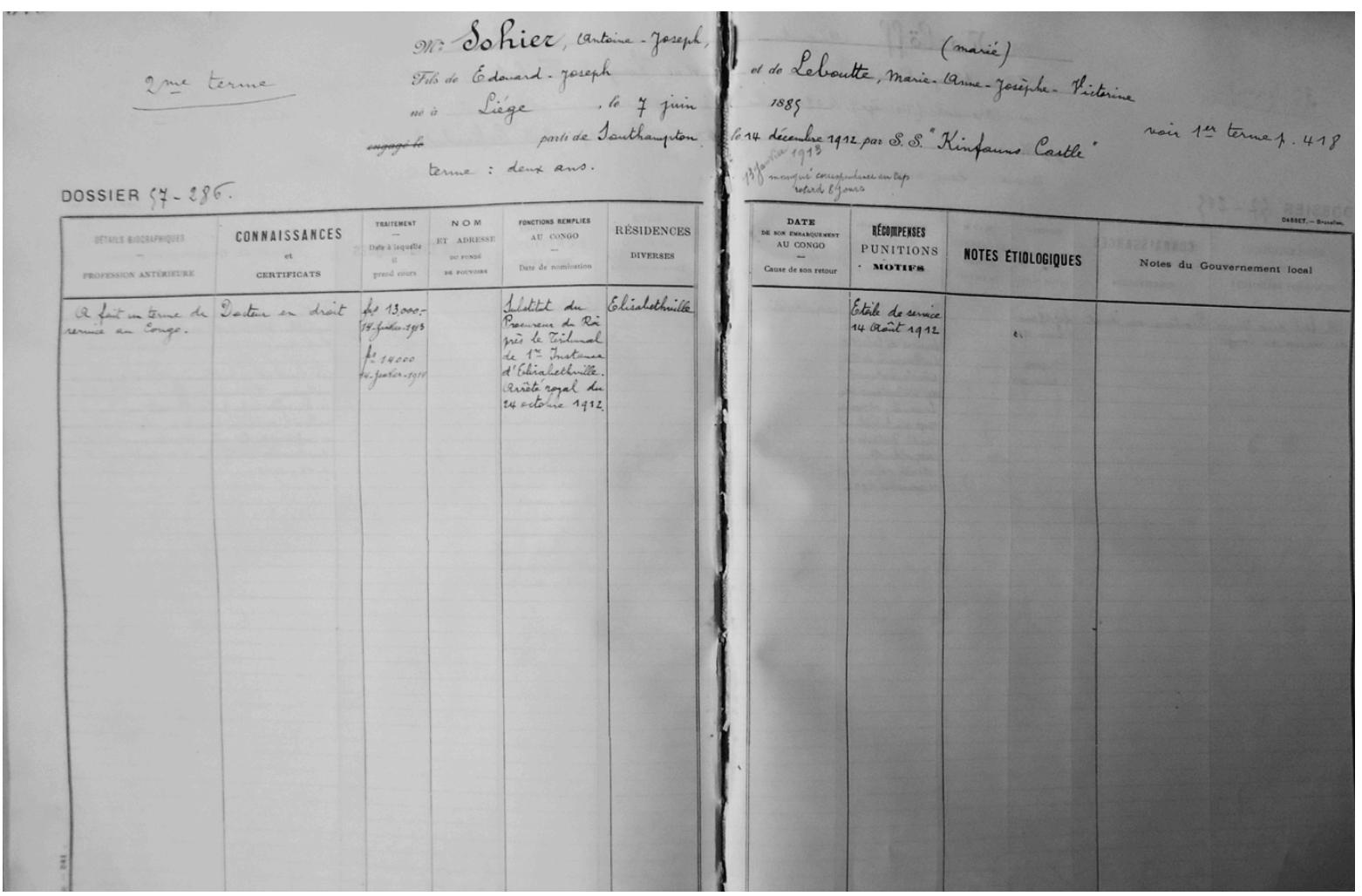

Fig. 1 : Fiche signalétique d'Antoine Sohier (deuxième terme) 19

À côté de cette source, les registres du service du personnel d'Afrique (registres SPA) font figure d'exceptions. Tenus à Bruxelles par le département des Affaires étrangères, ils livrent toute une gamme d'indications sur le personnel judiciaire, dont les magistrats $\underline{20}$. Ils pallient l'absence de la plupart des dossiers personnels des agents de l'ÉIC qui n'ont pas ou plus servi l'État après la passation de pouvoir à la Belgique. Ces registres se composent de fiches signalétiques d'une double page et couvrent un «terme » d'un agent21, ainsi que toute une série d'éléments qui permettent de reconstituer l'essentiel des carrières, avec leurs principales bifurcations et la nature des fonctions occupées22. Chaque nouvel engagement donnait lieu à l'ouverture, dans le registre en cours, d'une nouvelle fiche. Le nombre de fiches, pour un même agent, réparties sur un ou plusieurs registres, traduit une plus ou moins longue carrière au service de l'État indépendant. Fort heureusement, la reconstitution des carrières est facilitée par les repères introduits sur le bord droit des fiches par les commis du département : sur chaque fiche sont indiqués les numéros de registre et de fiche des autres engagements assurés par l'agent. La richesse de la source tient également aux données sociologiques qu'elles permettent de collecter et qui sont éclairantes sur le profil des magistrats recrutés : nationalité de l'agent, formation universitaire, profession avant son départ, adresse. Ces registres compilent aussi les décorations et les punitions des agents, ainsi que de brèves évaluations rédigées par leur hiérarchie. Ces dernières révèlent la force d'une évaluation sur le terrain dans le cadre d'un recrutement métropolitain classique fondé sur les qualités morales des agents, leur recommandation et un rapide entretien. À la fin de la période couverte, et en particulier dans le dernier volume (SPA 96), le contenu des registres tend à s'appauvrir. La colonne « notes » n'est plus systématiquement renseignée, et bien souvent on ne sait pas quelles furent les fonctions occupées au Congo par l'agent.

Particulièrement utile pour travailler sur les carrières des magistrats, l'ensemble des 
Prosopographie et biographie : regards croisés sur la magistrature coloniale ...

registres SPA n'est cependant pas adapté à l'élaboration d'une carte judiciaire et d'une pyramide des juridictions du Congo. Il a en effet pour objet non pas les tribunaux et les fonctions judiciaires qui leur sont associées mais le personnel. Comme on va le voir dans le point suivant, cela pose quelques difficultés, notamment lorsqu'il s'agit d'introduire de nouvelles données dans la base Belgian Magistrates.

\section{L’intégration systématique de données coloniales imprécises}

Lors de sa création en 2005, la base de données "Prosopographie des magistrats belges 1795-1960» (Belgian Magistrates) est conçue pour recevoir des données relatives à la magistrature métropolitaine, dans un cadre contraignant de saisie adapté à ces dernières. Larchitecture de la base impose d'indiquer les dates de création et de suppression de chaque juridiction et invite (sans obligation) à indiquer sa localisation, ses compétences, le régime politique durant lequel elle a existé, son ressort et enfin, sa place dans la pyramide judiciaire. Dès lors, lorsque l'intégration de données fut étendue à celles des juridictions et de la magistrature coloniales, plusieurs problèmes se sont posés - de nouvelles avancées allant souvent de pair avec de nouveaux défis techniques. En effet, la création d'une nouvelle fiche de magistrat est impossible si ne sont pas présentes, dans la base, les juridictions dans lesquelles ce magistrat a officié et les fonctions judiciaires attachées aux dites juridictions. Ces champs sont en effet subordonnés l'un à l'autreㄹ․ Chaque fiche représente une juridiction dans un environnement politique, spatial et temporel bien défini (ainsi, le passage de l'ÉIC à la colonie belge impose à priori de clore toutes les fiches de juridiction, pour en ouvrir de nouvelles). Or, sur le plan des juridictions, le cas colonial diffère du cas métropolitain. La carte judiciaire coloniale est plus flexible que la carte métropolitaine : de multiples facteurs président à un remaniement régulier des limites des juridictions, à commencer par les conditions d'occupation du territoire telles que la conquête, l'état de guerre ou de rébellion. $\mathrm{Au}$ début de ce projet, les recherches sont encore peu nombreuses, et la carte judiciaire n'est pas encore assurément et entièrement établie, du moins jusqu'en 192024. Le travail a pu aussi être compliqué par des formulations différentes, selon les sources. Et pour cause, les sources, et plus principalement les Bulletins officiels, restent insuffisantes pour saisir exhaustivement l'évolution de l'organisation judiciaire. Il arrive régulièrement, en effet, qu'un décret s'appuie sur, ou abolisse, des textes antérieurs dont on découvre à l'occasion l'existence25. De même, il n'est pas impossible que des juridictions transitoires semblables aient existé sans que ce soit consigné dans les sources imprimées. La difficulté provient aussi des changements de dénomination des tribunaux, dont les ressorts sont soumis à variation, notamment dans les premiers temps de la colonisation. De plus, ces juridictions ayant été soumises à une fluctuation incessante, que ce soit du point de vue de leurs ressorts, comme de leur siège - en tout cas au moins sous l'ÉIC, le travail de saisie des fiches personnelles a été d'autant plus fastidieux que les bifurcations administratives étaient nombreuses - et bien entendu non synchronisées avec les données personnelles. Ainsi, les publications officielles au Bulletin officiel et les registres SPA, à usage interne, ne contiennent pas systématiquement les mêmes identités juridictionnelles, de sorte qu'il a pu être difficile de trouver à quelles juridictions déjà présentes lier tel ou tel magistrat.

Dans le cadre du projet de recherches Belgafrican Magistrates, le fait de travailler d'abord sur les premiers temps de la colonisation a sans doute donné à la cartographie judiciaire une acuité particulière. En effet, puisque le remodelage territorial est constant en situation coloniale, selon l'état des finances de la colonie et de la métropole ou selon les enjeux éventuellement contradictoires du contrôle social et de l'affichage international, les juridictions destinées aux 
Européens, beaucoup plus lisibles et stables que celles qui visent les populations indigènes et les militaires, ont été préférées dans un premier temps. Dans le cas précis de l’ÉIC, vers la fin de ce régime, il est frappant par exemple de voir un arrêté formulé ainsi qu'il suit : "Arrêté du 14 août 1905, $\mathrm{n}^{0} 35$, supprimant le conseil de guerre d'Albertville (Toa) et rattachant le ressort de cette juridiction au conseil de guerre établi au chef-lieu du secteur du Tanganika-Moero »26. Comme on le voit, la localisation n'est pas mentionnée. Il faut probablement y voir le signe que le dit chef-lieu n'était pas établi de façon définitive et qu'il pouvait être déplacé. Avec une telle formulation, le pouvoir judiciaire était appelé à suivre le pouvoir administratif, sans qu'il faille prendre un nouvel arrêté. Il est tout aussi courant d'apprendre, par un long rapport au Roi Souverain sur « l'œuvre au Congo », qui figure au Bulletin officiel de 1891 et qui fait l'éloge d'une administration judiciaire venue «substituer le règne de la loi à l'anarchie »27, d'apprendre qu'une justice ordinaire fonctionne dans le Bas-Congo, «où l'autorité de l'État est consolidée », tandis que le HautCongo, au-delà du Stanley-Pool, est soumis à la justice militaire : «Il y a des conseils de guerre à Équateur-Ville, à Nouvelle-Anvers (Bangala), à Basoko (Arouwimi), aux Stanley-Falls, au Lomami, à Lousambo, à Loulouabourg, au Kuango oriental, à l'Ouellé et au Katanga »28. Or, dans ce cas précis, les décisions de création de ces juridictions ne figurent pas dans les Bulletins antérieurs, il est donc, de ce fait, bien difficile de déterminer les dates auxquelles certaines juridictions se sont mises à exister. Ce type d'indication, courant pour la justice militaire, a compliqué la tâche. Il faudrait en effet pouvoir suivre l'ensemble des variations administratives, ce qui n'a pu pour l'instant être fait. La question est cruciale lorsqu'il s'agit de mettre en relation d'une part ces données juridictionnelles, avec d'autre part, les données personnelles des agents.

L'autre principale difficulté à s'être posée lors de l'intégration des informations coloniales à la base de données est celle de la grande flexibilité des magistrats coloniaux belges, comme l'ensemble des agents coloniaux européens du reste. S'il est généralement possible d'identifier une nouvelle fonction attribuée à un magistrat en plus de sa charge - comme par exemple, l'exercice de l'État civil - on ne sait pas toujours quand cette fonction a pris fin. Ce qui implique que, de façon générale, l'encodage des carrières s'appuie sur les dates de nomination à telle ou telle fonction, mais peu sur les dates de fin de ces activités, sauf à supposer que toutes les fonctions cumulées en un lieu cessaient en même temps avec la mutation du magistrat dans une autre juridiction. Par ailleurs les fonctions dans l'ordre judiciaire sont pré-encodées dans la base. Mais les fonctions par intérim ou les postes de «faisant fonction » ne l'ont pas été jusqu'ici. Or ces données sont relativement importantes, puisque spécifiques à l'ordre judiciaire colonial. De plus, les nominations et les mutations de la période léopoldienne ne sont, en effet, que partiellement consignées dans les Bulletins officiels. Ces derniers se limitent en effet à rapporter scrupuleusement les nominations à la cour suprême, le Conseil supérieur du Congo - leur valeur honorifique y contribuant sans doute - et régulièrement, mais avec des imprécisions, les nominations aux tribunaux d'appel et de première instance du Bas-Congo. Ainsi dans le Bulletin officiel de 1889, il est question des nominations «par décrets des 9 mai et 22 juillet 1889 » de M. De Saegher comme juge du Tribunal de première instance du Bas-Congo, de M. Baerts, comme procureur d'État et de M. Van der Straeten comme juge du Tribunal de première instance à titre personnel, sans que l'on sache précisément quelle date rapporter à quel individu - élément qui compte dans l'alimentation d'une base de données. Enfin, l'exploitation des données coloniales a imposé une réflexion et des ajustements techniques. Par exemple, aucun champ ne permettait de faire la distinction entre les magistrats engagés dans une carrière métropolitaine et ceux engagés dans une carrière coloniale. Toute recherche ciblée sur l'une ou l'autre carrière s'en trouvait empêchée. Il est à présent techniquement possible de mettre en lumière les caractéristiques 
Prosopographie et biographie : regards croisés sur la magistrature coloniale ...

générales de la magistrature coloniale, avec à l'appui, des données quantitatives transversales.

\begin{tabular}{|c|c|c|c|c|c|}
\hline Référence & Personne & Juridiction & Nomination & Recherche SQL & Logout \\
\hline \multicolumn{6}{|c|}{ Prosopographie Rechercher une personne } \\
\hline
\end{tabular}

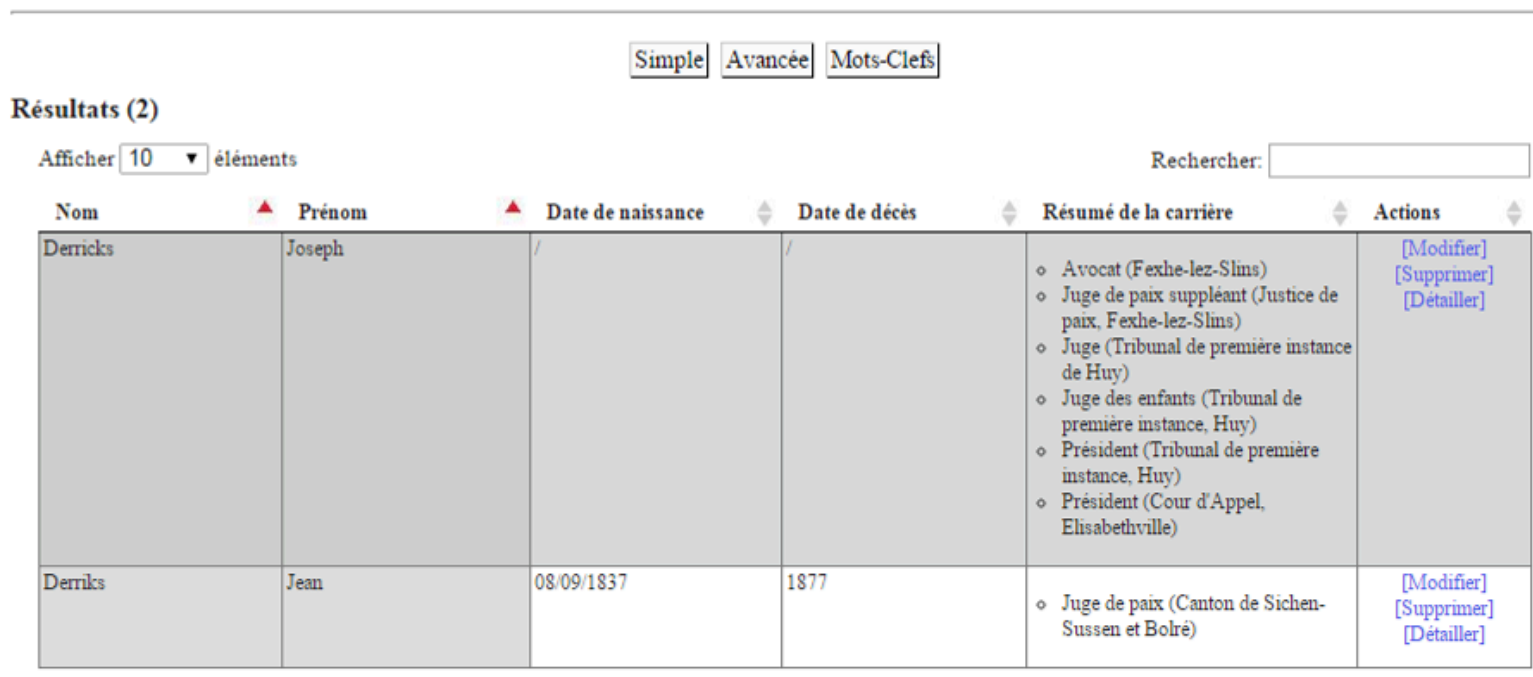

Affichage de l'élement 1 à 2 sur 2 éléments

Fig. 2. : Capture d'écran de la base de données « Prosopographie des magistrats belges ».

Rechercher une personne - Derriks

À l'heure actuelle, l'ensemble des informations officielles relatives aux juridictions et aux magistrats en fonction durant la période de l'ÉIC a été intégré à la base, soit 112 juridictions et près de 350 magistrats. Les premières manipulations de la base de données livrent déjà de riches enseignements concernant l'organisation de la magistrature congolaise durant l'époque léopoldienne et les premières années de la colonie. Il est dorénavant possible de dégager les principales tendances quant au recrutement, la formation et la carrière type d'un agent du corps judiciaire colonial belge pour ces périodes et ainsi préciser, confirmer ou nuancer, des éléments qui n'étaient jusqu'alors que des hypothèses pour le cas colonial belge telle la nationalisation du cadre administratif à partir de la reprise du Congo par la Belgique (1908)⒉ En outre, les premières manipulations de la base de données mettent en exergue des réseaux dont l'analyse doit être poursuivie afin d'en mesurer la portée et les implications. Parmi ceux-ci, citons le réseau liégeois dont font notamment partie Fernand Dellicour, Eugène Jungers, Octave Louwers et Antoine Sohier. Tous ces magistrats de premier ordre sont partis au Congo après avoir été formés et encouragés dans leur départ par le professeur Gérard Galopin. Un second réseau est né autour du Tribunal de première instance d'Élisabethville dès les années 1910. Principalement animé par Antoine Sohier et Joseph Derriks, il se concrétise par la Revue juridique du Katanga et la Revue des Juridictions indigènes ainsi que par la publication d'ouvrages de doctrine.

Continué depuis juillet 2014 par le projet Belgian Magistrates Social networks, les objectifs du projet se sont élargis à l'étude du profil collectif de la magistrature du Congo belge (1908-1960), et du Ruanda-Urundi sous mandat belge (1924-1962) et son analyse sous l'angle des réseaux sociaux par la mise en œuvre d'une série de travaux de recherche qui s'attachent, entre 
autres, à répondre à ces nouvelles questions : de quel milieu intellectuel et social les membres du corps judiciaire colonial sont-ils originaires ? Comment contribuent-ils par leurs publications à la formation d'un droit et d'une pensée juridique « congolais » ? Le projet de thèse d'Amandine Dumont (Université catholique de Louvain) analyse le corps judiciaire colonial belge par le prisme de leurs réseaux sociaux et intellectuels, tandis que les travaux de Pascaline le Polain de Waroux puis de Romain Landmeters (Université Saint-Louis - Bruxelles) adoptent une approche qui mêle la biographie et la prosopographie à l'étude des journaux et périodiques. Les acquis relatifs à la période de l'ÉIC, fondés sur le dépouillement des Bulletins officiels, des Recueils mensuels et des registres SPA notamment, pourront ainsi être prolongés et placés dans une plus large perspective lorsque l'encodage des données pour le Congo belge et le Ruanda-Urundi sera poursuivi.

\section{Antoine Sohier, du Tribunal de première instance de Lukafu à la Cour de cassation de Belgique}

Si l'analyse prosopographique ne s'intéresse normalement pas à l'unique, mais bien au commun et au général, elle appelle néanmoins à dégager les parcours singuliers et à mettre en évidence des carrières exceptionnelles telle celle d'Antoine Sohier. Ce dernier s'engage dans la carrière coloniale en 1912 ; il en atteint les sommets puisqu'il deviendra procureur général du Congo belge puis président de la Cour de cassation belge. Parallèlement à cette activité, il contribue à développer le champ des études juridiques en fondant plusieurs revues de doctrine et de jurisprudence coloniales dont il est l'un des premiers auteurs, abordant dans celles-ci une variété de thématiques. 


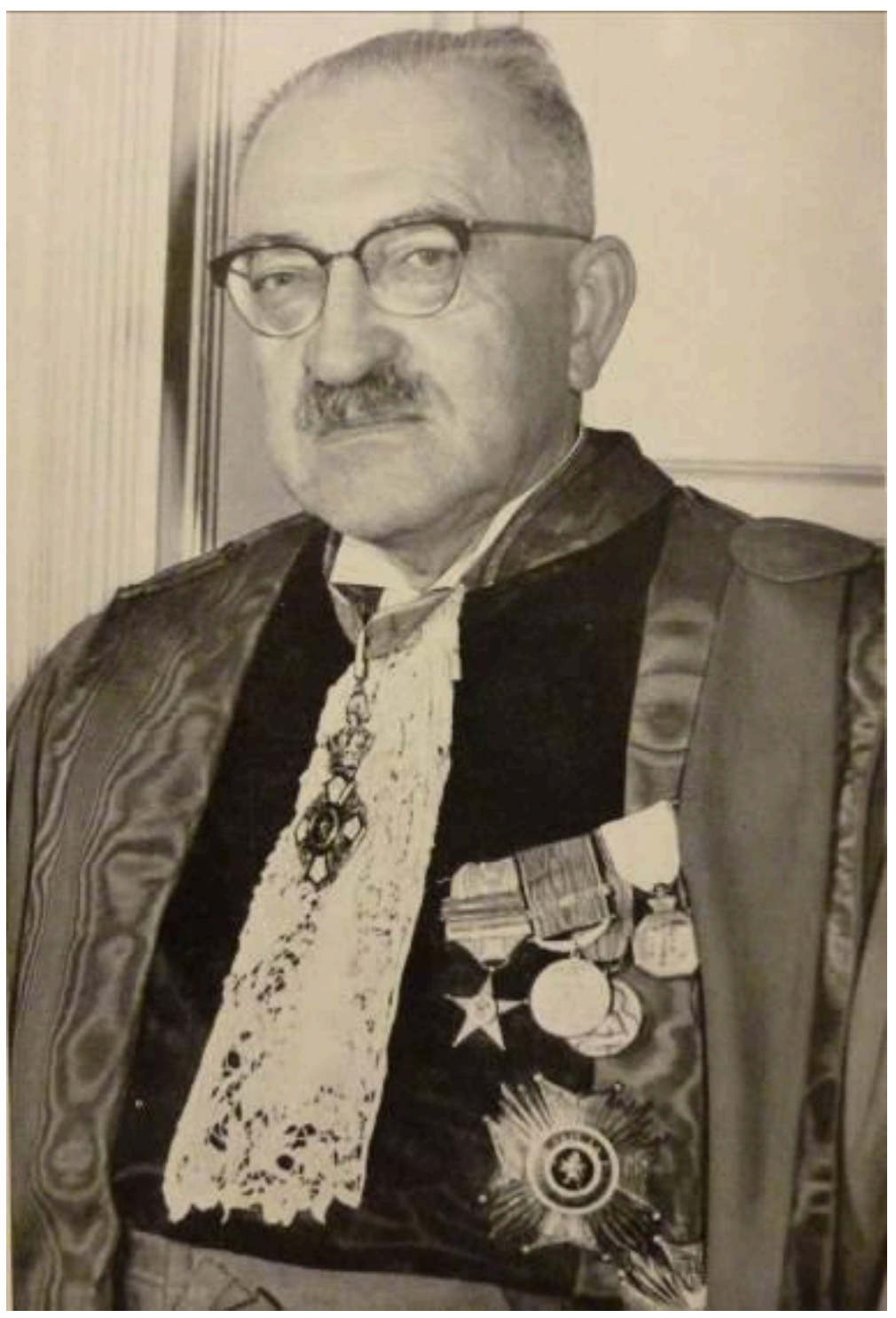

Fig. 3 : Antoine Sohier $\underline{30}$

\section{Catalyseur de la pratique et de la pensée juridique coloniale}

Né à Liège le 7 juin 1885, Antoine Sohier devient docteur en droit de l'université de sa ville natale en 1908. Suivant les traces de ses ainés sortis de la même faculté, tels Eugène Jungers, Octave Louwers ou Fernand Dellicour, il s'engage rapidement dans une carrière coloniale. Soutenu comme de nombreux anciens étudiants par son professeur Gérard Galopin31, Antoine Sohier décide, après un stage de dix-huit mois au barreau liégeois, de partir pour le Congo belge en qualité de magistrat32. Nommé substitut suppléant du procureur d’État le 10 mai 1910, il embarque quinze jours plus tard à Southampton sur le Kenilworth Castle vers sa nouvelle destination. Sohier n'a alors que vingt-deux ans, il fait partie de la vague de jeunes Belges partis nationaliser les rangs des magistrats coloniaux après la reprise de l’ÉIC par la Belgique $\underline{33}$.

Antoine Sohier arrive au Katanga le 8 juillet 1910. Il est accueilli par le procureur du roi Sigvald Meek $\underline{34}$, magistrat norvégien chargé de le former $\underline{35}$. Jeune magistrat, il commence sa carrière coloniale par un stage au Parquet de première instance de Lukafu et aux Parquets des 
districts qui en dépendent. Moins d'un an plus tard, en avril 1911, il est nommé juge suppléant du Tribunal de première instance de l'Étoile (qui deviendra par la suite Élisabethville) et substitut du Tribunal de Kiambi et du Conseil de guerre du Tanganika-Moëro. Ces premières attributions lui permettent de se rendre compte de la polyvalence et de la mobilité exigée des magistrats. Il clôture son stage après être devenu substitut du procureur du roi titulaire, le 14 octobre 1912. Du premier de ses sept termes, Antoine Sohier garde en mémoire les difficultés pour les magistrats d'assumer les lourdes tâches qui leur sont confiées alors qu'ils ne bénéficient d'aucune formation pratique adéquate. Il reviendra d'ailleurs sur cette question près de seize ans plus tard dans son discours prononcé à la conférence du Jeune Barreau de Bruxelles et dans lequel il déclare :

Une année et demie de barreau, consacrée surtout à des affaires commerciales, n'avait pu lui donner sur ses fonctions futures, poursuites, instruction, jugement que des notions vagues et théoriques. Or au poste-frontière, il reçut par lettre l'ordre de s'y arrêter, et quinze dossiers importants à instruire ; le même jour un homicide s'y commettait... Après quelques semaines, il gagnait enfin le siège du parquet et y travaillait pendant deux mois sous l'ordre du faisant fonction de Procureur du Roi. Il était ensuite envoyé en brousse pour l'instruction d'une affaire à laquelle le voisinage de la frontière avait donné une importance spéciale. Rappelé bientôt par une maladie du Procureur du roi, il gérait le parquet pendant cinq à six semaines avec notamment la charge de mettre au courant un magistrat récemment arrivé, dont il était déjà l'ancien. Le Procureur du roi rétabli, il est envoyé administrer un parquet de l'intérieur ; il s'y trouve isolé, à plus de mille kilomètres de tout autre magistrat... Il a presque toujours été seul, en correspondance avec ses chefs, mais les communications sont lentes, et à tout instant il doit prendre lui-même les décisions les plus importantes $\underline{36}$.

Le 14 décembre 1914, Antoine Sohier revient sur le sol congolais accompagné cette fois de son épouse Cécile Gulikers, dont il aura six enfants. C'est au cours de ce deuxième terme qu'il est nommé procureur du roi près le Tribunal de première instance d'Élisabethville, le 11 avril 1915. Tout au long de sa carrière, Antoine Sohier gravit les échelons des fonctions judiciaires ; le 5 décembre 1922, il y est nommé substitut du procureur général pour ensuite être élevé, le 15 mai 1925, à la fonction de procureur général d'Élisabethville. Il prend alors la relève de son collègue liégeois Fernand Dellicour, entré au service de l'État du Congo en 1906, et conserve cette fonction jusqu'au terme de sa carrière coloniale en 193437. Il se démarque en cela de ses nombreux confrères dont la carrière était faite d'itinérance. Après dix-huit ans de service, Antoine Sohier est au terme de sa carrière coloniale. Au moment de quitter le sol africain, il exprime l'émotion qu'il a de quitter ce qu'il qualifie de "grande famille judiciaire » et se remémore les difficultés, lorsqu'il était en congé, de se trouver au loin : « on sentait combien est grande cette fraternité qui lie les coloniaux; le jour de la séparation lui aussi montre l'intensité de tels liens $\$ \underline{38}$. Si ces liens sont effectivement tissés dans le milieu judiciaire colonial leur degré, leur nature et leur portée pourront être mis en exergue par une étude systématique des profils de la magistrature facilitée par l'étude des éléments introduits dans la base de données.

En septembre 1924, alors qu'il est substitut du procureur général au Katanga, Antoine Sohier, souhaitant combler « le manque d'esprit commun de la magistrature » $\underline{39}$, crée la Société d'Études Juridiques du Katanga dont il devient président. Cette association a pour but « d'étudier le droit colonial et de coopérer à son développement, d'aider au maintien des traditions des différentes professions judiciaires, et de resserrer les liens de confraternité entre leurs membres » $\underline{40}$. 
Prosopographie et biographie : regards croisés sur la magistrature coloniale ...

La même année, avec la collaboration du président de la Cour d'appel d’Élisabethville, Joseph Derriks, il fonde la Revue Juridique du Katanga. Vitrine de la Société éponyme, ce périodique a pour objectif «la gestion et le développement des publications juridiques » 41 . Par là, il cherche à fixer les principes juridiques et les coutumes en usage dans le ressort de la Cour d'appel d'une part et à les vulgariser de manière à faciliter les rapports d'ordre civil, commercial, administratif et social entre les habitants de la circonscription judiciaire d'autre part. Pour réaliser ces ambitions, la revue publie et commente la jurisprudence de la colonie ainsi que des études doctrinales $\underline{42}$. Qualifiée par le gouverneur général Martin Rutten de « redoutable instrument judiciaire»43, cette revue constitue un guide qui entend corriger et compléter ce que «la science du droit a de rudimentaire $\gg \underline{44}$. Signe de son expansion, elle devient, dès 1927, la Revue Juridique du Congo belge $\underline{45}$. Si l'efflorescence de ce périodique rend possible l'hypothèse de l'émergence de courants de pensée et d'une éventuelle école juridique katangaise, les milieux judiciaires ne sont pas les seuls concernés par ces publications. L'existence même de cette revue est en effet permise par le financement de compagnies industrielles telles que l'Union Minière ou la Compagnie foncière et le Comité Spécial du Katanga. Celles-ci coopèrent ainsi au développement du droit colonial jugé indispensable au fonctionnement de la colonie $\underline{46}$.

En 1933, soit peu avant son départ de la colonie, Antoine Sohier fonde le Bulletin des Juridictions Indigènes et du Droit Coutumier Congolais dont l'objectif est clairement annoncé dans le titre. Présentée comme le supplément à la Revue Juridique du Congo belge, cette publication a pour dessein de servir de guide aux centaines de juridictions créées par le décret du 23 octobre 192647. Dans la philosophie de son fondateur, cette revue défend l'idée que la connaissance des coutumes juridiques est nécessaire afin de surveiller et guider la construction du droit coutumier $\underline{48}$. Par la création de ce Bulletin, Antoine Sohier témoigne du rôle central qu'il attribue aux juridictions « indigènes » $\underline{4}$ pour l'administration et le maintien de l'ordre colonial mettant ainsi en évidence sa conviction en l'administration indirecte du Congo $\underline{50}$. Pour assurer la survie de ce périodique, le comité de rédaction mise sur la collaboration de magistrats et missionnaires de la colonie, dont les connaissances des mœurs et les expériences doivent nourrir les articles. Le soutien que reçoit ce bulletin témoigne une nouvelle fois qu'il répond à un besoin réel 151 . Celui-ci parait jusqu'en 1962, date à laquelle il est rattaché à la Revue Juridique.

Outre la création, gestion et animation de ces deux revues, Antoine Sohier collabore tout au long de sa carrière à des dizaines de périodiques parmi lesquels Congo : revue générale de la Colonie belge et Zaïre : revue congolaise. Auteur prolifique aux thèmes nombreux, la plupart de ses écrits sont traversés par la volonté de sensibiliser la magistrature au droit coutumier. Cet intérêt majeur qu'il développe prend tout son sens dans son " premier essai important relatif au droit coutumier » qu'il rédige en 1932 et intitule La pratique des juridictions indigènes 52 . Il met ainsi en exécution son leitmotiv : "se mettre à l'école du Noir pour apprendre son droit et ensuite le lui enseigner $" \underline{53}$. À travers l'attention qu'il porte à cette autre branche du droit colonial, Antoine Sohier met ainsi en lumière toute une série d'acteurs encore peu étudiés tels que les juges africains, de même qu'il met par écrit les questions coloniales de son temps.

\section{Une reconversion métropolitaine}

Malgré les craintes qu'il exprime quant aux possibilités de réintégrer la magistrature métropolitaine et d'y faire carrière, Antoine Sohier rentre en Belgique en 1934 afin d'y assurer la scolarité de ses enfants. En l'absence de garantie de reclassement dans le système judiciaire belge, 
il est contraint d'exercer une fonction inférieure à celle qu'il avait atteinte au Congo. Il entame ainsi sa seconde carrière le 11 juillet 1934, comme procureur du roi à Arlon avant d'être nommé conseiller à la Cour d'appel de Liège en 193754. Le 8 février 1946, il devient conseiller à la Cour de cassation. Après avoir assuré pendant douze ans cette fonction, il est appelé à l'unanimité en 1958 aux fonctions de président de chambre. Enfin, en mars 1960, il est nommé président de cette juridiction suprême $\underline{5}$.

Tout au long de sa carrière belge, Sohier se distingue à nouveau par ses multiples publications concernant le Congo comme la métropole $\underline{56}$. Au regard de ce que représente le Journal des tribunaux pour la métropole, il fonde en 1950 le Journal des tribunaux d'Outre-Mer dont il sera le rédacteur en chef jusqu'en 1961. Cet organe lui permet d'aborder toutes les problématiques juridiques et judiciaires du Congo belge. Il y publie régulièrement des chroniques sur la jurisprudence, la procédure civile et pénale et la coutume indigène. Il signe par ailleurs de nombreux articles dans d'autres périodiques tels que le Bulletin de l'Institut Royal Colonial belge, dont il est membre depuis 1930. À côté des revues, il met également en place une œuvre doctrinale qui se caractérise principalement par la synthèse de ses expériences et de sa connaissance des réalités coloniales. Parmi celles-ci comptent notamment un important chapitre sur les obligations et contrats en droit colonial dans les Novelles (1948), Le traité élémentaire de droit coutumier du Congo belge et la publication, avec divers collaborateurs, du Droit civil du Congo belge (1956)57. $\mathrm{Au}$ terme de sa vie, ce magistrat laisse par conséquent derrière lui une importante bibliographie qui est le reflet des valeurs et intérêts qui l'ont transporté tout au long de sa carrière $\underline{58}$.

Les liens qu'Antoine Sohier maintient avec la colonie belge ne se limitent pas à cette littérature spécialisée. Ils continuent d'animer sa vie professionnelle. Outre ses activités exclusivement juridiques et judiciaires, Antoine Sohier enseigne le droit coutumier à l'Institut universitaire des territoires d'Outre-Mer. Conscient du manque de formation pratique des futurs magistrats coloniaux, Sohier fonde ses cours sur des exemples puisés de son expérience personnelle모. Nommé membre du Conseil colonial60 en 1951, il participe à la commission consultative chargée, par l'arrêté royal du 24 juin 1955, d'étudier les réformes à apporter en matière de droit judiciaire au Congo belge. Par ailleurs, dans la période préalable à l'indépendance de la colonie, il est nommé au Conseil de législation dont la mission va s'étendre de 1959 à 1962. Il prend encore part aux travaux des sessions périodiques de l'Institut colonial international, de l'Institut des civilisations différentes et assume le poste de vice-président de l'Association internationale de droit africain (AIDA) établie et instituée à Paris depuis 1959. Enfin, trois de ses enfants s'engagent dans une carrière au Congo. Sont-ce ses nouvelles responsabilités qui le rendent plus prudent ? À partir de 1955 et jusqu'à sa mort survenue à Bruxelles le 22 novembre 1963, Antoine Sohier publie anonymement de nombreux articles sur la législation coloniale, le droit coutumier et la magistrature. On peut supposer que ses nouvelles attributions le rendent plus frileux à l'idée d'exposer ouvertement ses opinions.

\section{Les particularités de magistrature coloniale belge}

La carrière d'Antoine Sohier parait singulière à bien des égards. Son parcours s'inscrit néanmoins dans le sillon de celui des magistrats de l'ÉIC, et ce, malgré la reprise de l'État par la Belgique et les changements de structure que cela a impliqués. Son engagement au sein de la magistrature congolaise, la formation qu'il a suivie, sa trajectoire professionnelle se caractérisent en effet par les principales tendances dégagées à la suite de l'étude des archives du service du personnel d'Afrique. Cette analyse prosopographique, bien qu'elle ne se suffise pas à elle même, 
Prosopographie et biographie : regards croisés sur la magistrature coloniale ...

permet néanmoins d'éclairer une série d'aspects importants et donc de renforcer la connaissance de l'organisation du personnel judiciaire, tant du point de vue de son origine que de sa carrière. Mais surtout, elle permet de dépasser les expériences individuelles des agents de l'État, de sortir de la description factuelle et de donner un sens à l'ensemble du corps judiciaire colonial belge.

\section{Devenir magistrat}

La magistrature de l'État indépendant du Congo se distingue largement de la magistrature belge de la même époque par la nationalité des hommes qui la composent. À la différence de son homologue métropolitaine, elle compte en effet des non-Belges. Sur les quelque 350 magistrats actuellement recensés dans la base de données, on dénombre vingt Norvégiens, trois Danois et quinze Italiens. D'autres nations sont également représentées au sein de ce corps, en proportions moindres toutefois. On y trouve un Suédois, un Français, trois Suisses, deux Roumains et même un Américain, natif de Boston. Docteur en droit de l'université de Christiana (Oslo) et avocat dans la petite ville de Grimstad avant de s'engager au service de l'État indépendant du Congo, ce dernier peut néanmoins être rapproché des magistrats norvégiens $\underline{61}$. Loin d'être cantonnés à des postes subalternes, plusieurs parmi eux ont occupé les sièges les plus importants de l'ÉIC à l'instar de l'Italien Giacomo Nisco qui fut président du Tribunal d'appel de Boma avant de devenir consul général de l’État léopoldien en Italie.

Cette composition bigarrée de la magistrature congolaise est due au contexte politique national. Ne parvenant pas à gagner l'adhésion des Belges et à recruter les membres de son administration africaine parmi ses sujets, Léopold II est contraint de se tourner vers l'étranger. Il se dirige vers les «petits » pays dénués de projet colonial en Afrique subsaharienne et engage principalement des ressortissants scandinaves et italiens. Ceux-ci ont peuplé la magistrature, mais aussi l'administration centrale à Banana puis Boma (capitales successives de l'ÉIC), l'administration locale, et enfin la Force publique (la force armée du Congo). Placer certains de ces étrangers à des postes de premier plan tient aussi à la situation diplomatique difficile du Congo. Issu de la conférence internationale de Berlin de 1885, le jeune État est soumis au regard des grandes puissances colonisatrices et particulièrement exposé aux critiques. C'est le cas par exemple lors de l'affaire Stokes-Lothaire, en 1895-1896, qui pèse d'un poids tel que l'État réforme l'institution judiciaire. C'est dans ce contexte que le Tribunal d'appel qui se saisit de l'affaire est renforcé par deux conseillers, l'un Italien et l'autre Suédois. Le secrétaire d'État Edmond Van Eetvelde ne manque pas de le souligner, en précisant : «Il a paru opportun de composer ce Tribunal d'appel d'éléments appartenant à différentes nationalités $» \underline{62}$.

Lors de la reprise de l'État indépendant du Congo par la Belgique en 1908, le gouvernement colonial s'emploie à nationaliser les cadres de l'administration. Même si aucune loi n'impose que les nouveaux magistrats soient de nationalité belge, les nouvelles recrues le sont presque exclusivement. Les Rapports annuels sur l'administration du Congo belge permettent de suivre ce mouvement. Si un peu plus de $58 \%$ de magistrats sont belges en 1909, c'est le cas de $82 \%$ puis 89 \% des effectifs en 1911, 1913 et 1918. Le nombre d'étrangers présents dans la magistrature coloniale se réduit alors à quelques unités. Entre 1913 et 1918, il n’y a plus que quatre Italiens et quatre Norvégiens parmi les magistrats 63 . Il s'agit généralement d'immigrés ayant résidé en Belgique ou y ayant suivi leur formation. Citons notamment le Roumain Chirila Adreiu, devenu substitut du procureur du roi à Matadi en 1905, après avoir été avocat à Bruxelles64. En parallèle à cette nationalisation des cadres de l'administration, la politique de recrutement des années 1908 
et suivantes est caractérisée par une seconde tendance. Le recrutement des candidats pour le Congo se fait désormais parmi les civils alors que Léopold II avait valorisé les militaires. En effet, sous l'ÉIC et plus particulièrement durant les premières années du régime, les magistrats et les fonctionnaires étaient principalement issus de l'armée belge. Camille Coquilhat, par exemple, avait quitté ses fonctions d'adjoint d'État-major pour mener des missions de « pacification » du territoire congolais avant d'y devenir gouverneur général.

La magistrature coloniale se distingue également par le fait que la plupart des magistrats qui la composent sont très jeunes. Ils sortent à peine de l'université où ils ont obtenu leur doctorat en droit, ou ont déjà fait quelques années de barreau, à Bruxelles, à Charleroi ou à Liège. Le barreau de Liège, en particulier, a compté d'éminents magistrats coloniaux, dont Antoine Sohier. D'autres ont été employés par une administration - une commune, un ministère et le service des prisons notamment. D'autres encore ont été au service de l'administration du Congo. S'ils ont été formés au droit belge ou à un droit étranger, ils sont seulement une poignée à jouir d'une expérience antérieure dans la magistrature. Citons par exemple August Pedersen-Norlöff qui était juge suppléant près le tribunal de Skien (Norvège) avant de s'embarquer pour le Congo, Aurel Varlam qui occupait la même fonction à Vaslui (Roumanie) et le suédois Hermann Antell, nommé président du Tribunal d'appel en 1897, après avoir été président du Tribunal de première instance de Färs. Plusieurs d'entre eux auraient dès lors formé leurs jeunes confrères inexpérimentés, et notamment les Belges. Jacques de Lichtervelde fait figure d'exception, lui qui a mis un terme à sa carrière de juge près le Tribunal de première instance de Bruxelles pour devenir juge du Tribunal territorial de Stanleyville en 1906. Étant donné que les magistratures belge et congolaise sont, à ce moment, des entités autonomes, en quittant la Belgique il a dû tirer un trait sur sa carrière bruxelloise et renoncer à sa pension $\underline{65}$.

En raison de leur jeune âge, la plupart des hommes devenus magistrats coloniaux sous l'ÉIC n'ont donc aucune expérience de l'administration de la justice ou du Congo. Ils se familiarisent avec ces questions en lisant les rares ouvrages qui y sont consacrés dont État indépendant du Congo. Historique, géographie, physique, ethnographie, situation économique, organisation politique d'Alphonse-Jules Wauters. Leur formation se fait par conséquent essentiellement sur le terrain. À partir du décret du 21 avril 1896, il est demandé aux nouveaux engagés d'accomplir un stage de deux ans et demi $\underline{66}$. Nommés à titre provisoire, les candidats magistrats occupent alors les fonctions de juge suppléant ou de substitut suppléant et exercent leur mission sous le contrôle d'un " ancien ». Toutefois, par manque de personnel, cet écolage se réduit fréquemment à peu de choses. Dans la plupart des cas, dès le lendemain de leur arrivée au Congo, les jeunes magistrats se trouvent en effet seuls à la tête d'un parquet ou d'une juridiction. Rentrant en congé en Belgique à la fin de leur stage, les magistrats ont encore à présenter un mémoire67 sur un sujet de droit ou de législation spécifique au Congo, avant d’être nommés définitivement68.

À la reprise du Congo par la Belgique, le stage et la défense du mémoire en droit colonial restent d'actualité. Toutefois, à cette formation de terrain s'ajoute dès 1911, avec l'ouverture de la section juridique de l'École coloniale de Bruxelles $\underline{69}$, une formation juridique spécifique, préparant à la magistrature coloniale belge. Cette formation d'environ quatre mois, confiée à des praticiens du droit généralement issus du ministère des Colonies, s'articule alors autour des cours de " Droit public et administratif du Congo», d'« Organisation et compétence judiciaire au Congo», de « Droit civil et pénal congolais » et de « Procédure civile et pénale congolaise » $\underline{70}$.

Enfin, la promulgation de la loi du 10 août 1921 permettant aux magistrats belges partis 
Prosopographie et biographie : regards croisés sur la magistrature coloniale ...

pour le Congo de conserver leur ancienneté et leurs titres à l'avancement favorisera le départ de magistrats expérimentés comme Joseph Derriks qui quitte la présidence du Tribunal de première instance de Huy pour celle de la Cour d'appel d'Élisabethville dès 1924.

\section{Faire carrière}

Les carrières africaines ne peuvent nullement être comparées à celles menées en métropole. Si entrer dans la magistrature belge signifie s'engager dans une carrière assurée et définitive, il n'en va pas de même en ce qui concerne la magistrature coloniale. Les magistrats n'y font, en moyenne, que deux à trois termes. Ils quittent ensuite l'Afrique et les rudes conditions physiques (maladies, climat, etc.) et morales (éloignement des proches, privation de tout milieu intellectuel, etc.) qu'elle leur impose. C'est en raison de ces éléments que la carrière coloniale est considérée comme complète au terme de dix-huit ans de service 71 .

Lors de leur premier terme, les magistrats sont préférentiellement affectés à Boma ou non loin de la capitale afin d'être supervisés de près par leurs supérieurs. Durant leurs termes suivants, ils sont envoyés dans des postes plus lointains et occupent des fonctions diverses - la recherche des infractions, leur jugement, l'exécution de ceux-ci, l'administration du parquet72. Au fil de leur carrière, les magistrats du Congo occupent des postes divers, passant de la fonction assise à la fonction debout, de la justice civile à la justice militaire, voire menant les deux de front. Le premier terme de Charles Smets illustre très bien cela :

Charles Smets, jeune docteur en droit, entre au service de l'État indépendant en qualité de magistrat à titre provisoire [...]. Il allait, comme la plupart de ses collègues, passer un premier terme de service dans une itinérance constante. Attaché à son arrivée à Boma, par un arrêté du Gouverneur général du 27 août 1904 - il avait été nommé le 4 août - en qualité de substitut suppléant au parquet du Tribunal de première instance unique de l'État, il serait, dès le 12 janvier 1905, attaché comme substitut suppléant au Tribunal territorial et au conseil de guerre de Coquilhatville, rappelé au parquet du Tribunal de première instance par arrêté du 13 novembre suivant, puis par un arrêté du 19 janvier 1906, attaché comme substitut suppléant, toujours, au Tribunal territorial et au Conseil de guerre établis à Basankusu, chef-lieu de la zone de la Maringa-Lopori, région dont l'exploitation concédée à l'Anglo-Belgian India Rubber Company avait retenu de la part de la Commission d'Enquête une attention toute particulièrepz3.

Outre ces éléments communs à de nombreuses carrières coloniales, les magistrats du Congo exercent leurs fonctions dans des conditions éprouvantes. En raison du manque chronique de personnel, ils sont contraints d'assurer seuls le travail qui incomberait normalement à plusieurs personnes. Mais, surtout, les dispositions légales encadrant leur activité n'assurent pas, contrairement à celles prises en Belgique, leur inamovibilité. Les magistrats nommés à titre provisoire peuvent être déplacés, sans leur consentement, dans n'importe quelle circonscription du siège auquel ils sont attachés voire dans n'importe quelle localité du Congo si des besoins urgents l'exigent. Même les magistrats nommés à titre définitif peuvent être déplacés. Toutefois, il faut que des besoins urgents l'exigent, et la mesure ne peut être que provisoire4․ Le personnel judiciaire du Congo est donc soumis à une grande mobilité. Généralement, les agents cumulent également diverses fonctions, et étaient appelés à remplacer des collègues malades ou en congé - c'est la question fameuse de l'intérim, ou des postes de «faisant fonction». Ainsi, entre la mi-février 1907 
et avril 1909, soit en à peine deux ans, Magnus Falkenberg a occupé successivement les fonctions de substitut du procureur d'État à Boma, de substitut du procureur du roi près le Tribunal territorial de Bomokandi et du Conseil de guerre du même lieu avant de reprendre la tenue de substitut du procureur d'État à Nyangara puis celle de substitut du procureur du roi près le conseil de guerre de la zone de la Gurba-Dungu et de l'enclave de Lado.

\section{Revenir du Congo}

Généralement, les magistrats coloniaux accèdent à la pension vers cinquante ans. Toutefois, vu leur (jeune) âge et le montant de la pension qui leur est versée75, la plupart d'entre eux entament une seconde carrière à leur retour du Congo. Celle-ci suit alors une trajectoire particulière selon la période à laquelle intervient la mise à la retraite des magistrats.

En effet, parmi les magistrats dont la carrière prend fin durant le régime léopoldien, plusieurs occupent ensuite des fonctions dans l'administration centrale du Congo dont les bureaux sont établis à Bruxelles, à proximité du Palais royal. Adolphe de Cuvelier est de ceux-là. Précédemment juge du Tribunal d'appel à Boma, il est nommé directeur du département Justice en 1886. Quelque vingt années plus tard, Albrecht Gohr connait une trajectoire similaire puisqu'il est nommé à la tête de ce même département en 1905. Il devient ensuite président du Comité Spécial du Katanga. Camille Janssens est, quant à lui, désigné secrétaire général du département Finances en 1892, après avoir été juge du Tribunal de première instance de Boma. Parallèlement à ces activités, de nombreux magistrats honoraires sont nommés dans le conseil d'administration de sociétés et lobbys créés par Léopold II ou son entourage dont la Compagnie du Congo pour le Commerce et l'Industrie (C.C.C.I) et l'Intertropical-Comfina.

$\mathrm{Au}$ lendemain de la reprise belge par contre, les autorités coloniales veulent rompre avec les modes de gestion de l'ÉIC et tentent d'instituer une nouvelle façon d'administrer le Congo. Les anciens magistrats ne sont plus réintégrés dans l'administration centrale. Ils se dirigent alors vers la magistrature belge. Ils parviennent à faire valoir leurs compétences et leur expérience antérieure et se voient confier des sièges importants à l'image de Fernand Waleffe, anciennement juge du Tribunal d'État de Matadi, devenu conseiller puis président à la Cour de cassation. Ernest Dupont, qui finit sa carrière coloniale comme juge au Tribunal d'appel de Boma en 1909, devient à son retour juge près le Tribunal de première instance de Mons.

En revanche, les magistrats revenus en Belgique au terme de la Première Guerre mondiale parviennent quant à eux plus difficilement à faire carrière dans la magistrature belge. Ceux qui y accèdent sont désignés pour des postes inférieurs. C'est ainsi qu'Iwan Grenade, procureur d'État au Congo, devient juge de paix à Stavelot en 1920. Il n'est pas le seul à connaitre cette situation, si l'on se fie à Antoine Sohier :

Les uns voient leurs candidatures repoussées et leur expérience est perdue, d'autres parviennent à se faire nommer, mais le plus souvent sans qu'il soit tenu compte de leur ancienneté. Ils entrent par la petite porte aux postes inférieurs. On voit d'anciens substituts du procureur général ou des procureurs du roi coloniaux devenir en Belgique substitut de complément du procureur du roi ou substitut de l'auditeur militaire ; des conseillers de cour d'appel devenir référendaires, des présidents de cour, après vingt ans de colonie, figurer dans l'annuaire au bas du tableau des juges d'un Tribunal de première instance $\underline{76}$. 
Prosopographie et biographie : regards croisés sur la magistrature coloniale ...

De nombreux magistrats revenus du Congo dans l'entre-deux-guerres changent dès lors d'orientation professionnelle. S'il est particulièrement malaisé de suivre leur reconversion, il est à noter que plusieurs d'entre eux comme Fernand Dellicour - ancien procureur général - sont devenus professeurs. Ils dispensent les cours consacrés au droit et aux institutions du Congo aux étudiants des universités belges ou à ceux de l’École coloniale. Ils assurent ainsi une véritable formation aux futurs magistrats du Congo belge. Tandis que plusieurs magistrats honoraires du Congo, reconnus par leurs pairs métropolitains grâce à leurs nombreuses publications et les liens personnels qu'ils ont tissés tout au long de leur carrière coloniale, se démarquent de cette trajectoire en poursuivant une brillante carrière dans la magistrature belge. Ainsi, Jean De Muylder est devenu conseiller à la Cour d'appel de Bruxelles et Antoine Sohier a été nommé, nous l'avons dit, président de la Cour de cassation.

\section{Conclusion}

Quinze ans après le renouveau des études coloniales, il est apparu nécessaire de mettre en exergue l'appareil étatique des territoires ultramarins belges, dont la justice. L'opportunité de traiter de ce sujet n'a toutefois d'égal que sa complexité. Écrire l'histoire des institutions judiciaires et de la magistrature coloniales nécessite en effet de compulser des sources difficilement accessibles. Nombre d'entre elles ne sont en effet ni classées ni inventoriées $\underline{77}$. En outre, leur consultation est soumise à l'autorisation d'une instance tierce, la Commission de la protection de la vie privée. Par ailleurs, certaines de ces ressources documentaires, dont les registres du Service du personnel d'Afrique font partie, ne permettent pas de saisir toute la complexité de l'administration de la justice. Ainsi, celles-ci ne distinguent par exemple pas la date de nomination et de la prise de fonction effective. De la même manière, elles ne renseignent nullement les déplacements multiples et l'itinérance qui est imposée à de nombreux magistrats.

Il est possible de dépasser ces contraintes archivistiques grâce à la conjugaison de deux approches historiques : la biographie et la prosopographie. La première, plus classique, permet de mettre en exergue des carrières exceptionnelles comme celles de celui qui peut être considéré comme le catalyseur de la magistrature et de la science juridique coloniale - Antoine Sohier. La seconde approche, la prosopographie, ambitionne de dépasser l'expérience individuelle afin de dégager les lignes de force de la carrière des magistrats coloniaux.

Dans le cas présent, cette biographie collective est réalisée à partir d'une base de données conçue pour l'étude de la magistrature belge métropolitaine. Étendre le champ de cet outil aux espaces ultramarins permet donc d'étudier les institutions judiciaires métropolitaines et coloniales ainsi que leurs acteurs en vue de définir les uns et les autres, mais aussi et surtout, de questionner les relations entre les deux ensembles. Cette évolution de la base de données offre l'occasion de saisir l'organisation judiciaire instituée durant les premières années de la colonisation belge. Elle offre également l'occasion de renforcer la connaissance du personnel judiciaire, tant du point de vue de son origine géographique et sociale, des procédures de recrutement qui lui sont imposées, des principales étapes de sa carrière comme de la fin de celle-ci. En outre, en permettant de dépasser la simple expérience individuelle, l'approche prosopographique ouvre la voie vers de nouvelles pistes et hypothèses de recherche. À l'exemple du parcours d'Antoine Sohier, l'usage de ces nouveaux outils facilite la mise en exergue de carrières particulières qui peuvent, à leur tour, être étudiées plus en profondeur, que ce soit par le prisme des réseaux, de la biographie ou encore de l'analyse de leurs productions intellectuelles. Ainsi, l'étude du parcours professionnel qui le conduit du Tribunal 
de première instance de Lukafu à la présidence de la Cour de cassation renforce la connaissance des multiples échelons de la carrière judiciaire, mais surtout de mesurer la réalité de l'exercice de ces fonctions tandis que l'étude de l'œuvre scientifique et littéraire qu'il lègue permet par ailleurs de cerner à quel point il est un des fers de lance de la recherche et de la dissémination du savoir juridique, colonial et coutumier.

La recherche concernant la magistrature coloniale n'en est qu'à ses débuts. Il est aujourd'hui nécessaire de poursuivre les hypothèses formulées par la présente recherche. De la même manière, il faudra étendre le champ de la base de données au Ruanda-Urundi ainsi que revoir la définition des juges coloniaux afin de prendre en considération l'ensemble des personnalités investies du pouvoir d'instruction et de poursuite. Au premier rang de celles-ci figurent les membres de l'administration coloniale que sont les commissaires de district et les agents territoriaux. Ces nouvelles perspectives de recherche devraient aussi permettre de cerner avec davantage de précision les influences et emprunts de et vers la magistrature métropolitaine comme les autres organisations judiciaires coloniales, notamment française et britannique.

\section{Notes}

1 Cet article a été écrit dans le cadre du PAI 7/22 « Justice \& Populations : The Belgian Experience in International Perspective, 1795-2015 ». Une précédente version en a été publiée dans A. Hondeghem, X. Rousseaux et F. Schoenaers, Modernisation of the Criminal Justice Chain and the Judicial System. New Insights on Trust, Cooperation and Human Capital, Springer, 2016, p. 211-231. Le présent article présente des développements significatifs inédits.

2 Voir notamment P. VAN SCHUYLENBERGH, Trop plein de mémoires, vide d'histoire ? Historiographie et passé colonial belge en Afrique centrale, dans L'Afrique belge aux XIX ${ }^{e}$ et XX ${ }^{e}$ siècles. Nouvelles recherches et perspectives en histoire coloniale, éd.P. VAN SCHUYLENBERGH, C. LANNEAU et P.-L. Plasman, Bruxelles, P.I.E. Lang, 2014, p. 44 ; G. VANTHEMSCHE, De historiographie van het Belgische kolonialisme in Congo, dans De tuin van heden. Dertig jaar wetenschappelijk onderzoek over de hedendaagse Belgische samenleving, éd. G. VANTHEMSCHE, M. DE MeTSENAERE et J. BRugelman, Bruxelles, Vrije Universiteit Brussel Press, 2007, p. 417-456.

3 X. RousseauX, Vers une histoire post-coloniale de la justice et du droit en situation coloniale ?, dans Droit et justice en Afrique coloniale : tradition, production et réformes, éd.C. BRAILLON, L. Montel, B. Piret et P.-L. Plasman, Bruxelles, Université Saint-Louis - Bruxelles, 2014, p. 15.

4 À ce sujet, lire notamment : S. El Mechat (éd.), Les administrations coloniales XIX $X^{e}$-XX $X^{e}$ siècles. Esquisse d'une histoire comparée, Presses universitaires de Rennes, 2009 et D. M. ANDERSON et D. KilLingray (éd.), Policing the Empire. Government, authority and contrôle, 1830-1940, Manchester/New-York, Manchester University Press, 1991.

$\underline{5}$ M. FABRE, Le magistrat Outre-mer, un élément capital de la stratégie coloniale, dans La Justice et le droit : instruments d'une stratégie coloniale, Rapport fait à la mission de Recherche Droit et Justice, 2001 ; M. FABRE, Les justices coloniales : clones imparfaits du système judiciaire métropolitain, dans Quaderni Fiorentini, n ${ }^{0}$ 33/34, t. II, 2005 ; B. DURAND, Le Parquet et la Brousse. Procureurs généraux et Ministère public dans les colonies françaises sous la Troisième République, dans Staatsanwaltschaft, Europaische und Amerikanische Geschichten, Francfort-surle-Main, Max Planck Institut, Klosterman, 2005, p. 105-137 ; F. RENUCCI (éd.), Dictionnaire des 
Prosopographie et biographie : regards croisés sur la magistrature coloniale ...

juristes ultramarins (XVIII ${ }^{e}$-XX $X^{e}$ siècles), rapport broché édité au GIP Droit et Justice, 2012 ; J.-C. FARCY, Quelques données statistiques sur la magistrature coloniale française (1837-1987), dans Clio@Themis, $\mathrm{n}^{0} 4$, mars 2011.

6 P.-L. Plasman, Un État de non-droit ? L'établissement du pouvoir judiciaire au Congo léopoldien 1885-1889, dans op. cit., éd. C. Braillon, L. Montel, B. Piret et P.-L. Plasman, p. 27-28.

7 Nous entendons le vocable « colonie » et ses dérivés dans leur sens générique (et non juridique) ; elle nous apparait comme un territoire placé sous le contrôle d'une autorité étrangère. Ces termes s'appliquent dès lors tant au Congo belge qu'à l'État indépendant du Congo ainsi qu'au Ruanda et à l'Urundi alors que le premier n'est, formellement, dépendant d'aucun autre pays et que la Belgique administre les seconds en vertu d'un mandat confié par la Société des Nations (1924-1945) puis par l'Organisation des Nations Unies (1945-1962).

8 X. Rousseaux, op. cit., p. 15.

9 L. MONTEL, Le contrôle des magistrats dans le Congo léopoldien, d'après les registres du Service du personnel d'Afrique (SPA) (1885-1908), dans op. cit., éd. C. Braillon, L. MonTEL, B. Piret et P.-L. Plasman, p. 51-78.

10 P.-L. Plasman, Un État de non-droit ?..., op. cit., p. 37 ; B. PIRET, Les structures judiciaires « européennes » du Congo belge. Essai de synthèse, dans Ibid., p. 163-178.

11 En s'appuyant l'exemple colonial, la métropole adopte notamment le recours au juge unique après la Première Guerre mondiale (Loi du 25 octobre 1919 modifiant temporairement l'organisation judiciaire et la procédure devant les cours et tribunaux, dans Moniteur belge, 9 novembre 1919).

12 Recueil mensuel des ordonnances, arrêtés, circulaires, instructions et ordres de service, État indépendant du Congo, Boma, conservé à la bibliothèque du Service public fédéral Affaires étrangères, Commerce extérieur et Coopération au développement (SPF AE), à Bruxelles.

13 Tels que : A. LYCOPS et G. TOUCHARD, État indépendant du Congo ; recueil usuel de la législation des conventions internationales et des documents administratifs avec des notes de concordance, t. 1-7, Bruxelles, P. Weissenbruch, 1903-1913 ; O. LOUWERs, Lois en vigueur dans l'État indépendant du Congo. Textes annotés d'après les instructions officielles et la jurisprudence des tribunaux, Bruxelles, P. Weissenbruch, 1905. Remaniés sous le titre Codes et Lois du Congo belge puis actualisés par Pierre Piron et Jacques Devos (1943-1960), ces recueils resteront des références jusqu'à l’Indépendance congolaise de 1960.

14 L'exemple qu'offre le dossier individuel d'Edgard Vanderschueren fait regretter l'absence des autres. Cependant, il apporte un éclairage indispensable sur les registres du personnel d'Afrique, en particulier parce qu'on saisit d'autant mieux, grâce à lui, comment et pourquoi ces derniers furent produits et tenus. Dossier personnel d'Edgard Vanderschueren, SPF AE, AA, SPA 162, n ${ }^{0}$ 57-35; L. MONTEL, op. cit., p. 55.

15 É. CRUYSMANS, Oubliez-moi ! Droit à l'oubli, déréférencement, anonymisation et archives numériques, dans Human Rights as basis for Re-evaluating and Reconstructing the Law, éd. A. 
Hoc, S. WatTier et G. Willems, Louvain-la-Neuve, Bruylant, 2015, p. 403-418 et É. Dechenaud, Le droit à l'oubli numérique : données nominatives - approche comparée, Bruxelles, Larcier, 2015.

16 Bruxelles, SPF AE, Archives africaines (AA), Décrets de l'État indépendant du Congo non publiés au Bulletin officiel, $2 \mathrm{t}$.

17 SPF AE, AA, fonds ÉIC, L1 4885, Arrêtés non publiés 1885-1889, n 27 bis.

18 Par exemple, un registre du personnel judiciaire en congé est conservé, mais il ne couvre que les années 1947-1952. Les arrêtés royaux et ministériels de nomination du personnel conservés dans les archives du Service du Personnel d'Afrique, de même que les ordonnances du gouvernement local, ne sont par exemple pas conservés en série continue. SPF AE, AA, fonds Service du Personnel d'Afrique, liasses 195 à 232.

19 SPF AE, AA, fonds Service du Personnel d'Afrique, registre 892, p. 579.

20 Les «notes » sont scrupuleusement consignées jusqu'en 1912. Leur tenue se fait plus brouillonne ensuite. Elles sont abandonnées en 1914. L. MONTEL, op. cit., p. 52.

21 À partir du milieu des années 1920, l'ensemble de la carrière des agents coloniaux sera consigné sur une seule fiche signalétique. Le cas échéant, les différents termes réalisés seront renseignés successivement sur ce document.

22 Les fiches sont formées d'un tableau de dix colonnes, précédé d'un en-tête destiné à l'identification de l'agent. Elles contiennent les informations suivantes, indispensables à l'alimentation de la base prosopographique : nom, prénoms, date de naissance, situation familiale et filiation de l'individu, dates de début de terme, de nomination aux principales fonctions exercées, et de mutation, date de rembarquement pour la métropole en vue de la période de congé, voire s'il y a lieu, le motif et la date du décès (utile pour calculer le taux de décès en exercice et le comparer au taux métropolitain).

23 Contraindre la saisie de ces informations, en interdisant l'indication libre de la fonction et de la juridiction, satisfait aux principes des systèmes de gestion des bases de données selon lesquels l'information doit être standardisée pour pouvoir être ensuite traitée automatiquement. En contraignant la saisie, on réduit l'ampleur du nettoyage des données. Le choix était d'autant plus légitime que la saisie a toujours été faite à plusieurs mains. À ce sujet, lire notamment A. FRANÇOIS et F. MULLER, La prosopographie à l'heure du numérique : bilan, prospectives et perspectives à l'aune de l'application «Belgian Magistrates », publié dans ce volume.

24 Depuis lors, la thèse de doctorat inédite de Bérengère Piret permet notamment d'appréhender la carte judiciaire du Congo et du Ruanda-Urundi des années 1920 à 1960. B. PIRET, La justice coloniale en procès. Organisation et pratique judiciaire, le tribunal de district de Stanleyville (1935-1955), thèse de doctorat inédite, Université Saint-Louis - Bruxelles, 2016.

25 Par exemple : le décret du 27 avril 1889 sur la réorganisation de la justice répressive débute par « Revu le décret du 8 avril 1889 », qui n’est pas consigné dans le volume. De la même façon, le Bulletin officiel de l'ÉIC de 1897, dans son article $\mathrm{n}^{0} 1$, mentionne les rapports de l'arrêté du 5 août 1892, créant un tribunal territorial à Matadi, et de celui du 14 avril 1894, étendant au district des Cataractes la compétence du tribunal territorial de Matadi : ces deux arrêtés ne figurent pas 
Prosopographie et biographie : regards croisés sur la magistrature coloniale ...

dans les Bulletins officiels en question.

26 Recueil mensuel des Arrêtés, Circulaires, Instructions et Ordres de service, Boma, Imprimerie de l’État, 1905, p. 142.

27 Rapport au roi-souverain sur l'œuvre au Congo, dans Bulletin officiel de l'État indépendant du Congo,1891, p. 171.

$28 I d .$, p. 172.

29 L. H. Gann et P. Duignan, The Rulers of Belgian Africa 1884-1914, Princeton, Princeton University Press, p. 164-181.

30 J.-M. JADOT, Notice nécrologique : Antoine Sohier, dans Bulletin des séances de l'ARSOM, 1964 - fascicule 1, p. 169.

31 F. DeLLICour, Gérard Galopin, dans Biographie coloniale belge, t. IV, Bruxelles, Institut royal Colonial belge, 1955, col. 328-331.

32 A. SOHIER, Un début de carrière judiciaire. Souvenirs et réflexions, dans Journal des Tribunaux d'Outre-Mer, $\mathrm{n}^{0}$ 100, 15 octobre 1958, p. 145.

33 SPF AE, AA, fonds Service du Personnel d'Afrique, registre 895.

34 J. SOHIER, Sigvald Meek, dans Biographie coloniale belge, t. VIII, Bruxelles, Académie royale des Sciences d'Outre-mer, 1998, col. 283-288.

35 Chronique : dans le monde judiciaire, dans Revue de droit et de jurisprudence du Katanga, $\mathrm{n}^{0}$ 9, 15 juillet 1925, p. 254.

36 A. SOHIER, Les rapports entre les magistratures coloniale et métropolitaine..., op. cit., p. 11-12.

37 Chronique : dans le monde judiciaire..., op. cit., p. 255.

38 Chronique : le départ de monsieur le procureur général Sohier, dans Revue de droit et de jurisprudence du Katanga, $\mathrm{n}^{0}$ 2, 15 février 1932, p. 68.

39 Ces critiques sont notamment présentes dans : A. SOHIER, Les rapports entre les magistratures coloniale et métropolitaine..., op. cit., p. 13-14.

40 Société d'études juridiques du Katanga - Statuts, dans Revue de droit et de jurisprudence du Katanga, $\mathrm{n}^{0}$ 4, 15 février 1925, p. 102.

41 É. LAMY, Antoine Sohier, dans Biographie belge d'Outre-Mer, t. VIII, Bruxelles, Académie royale des Sciences d'Outre-mer, 1998, col. 392.

$\underline{42}$ Éditorial..., op. cit., p. 1.

43 Société d'Études juridiques. Fête du premier anniversaire..., op. cit., p. 53.

$4 \underline{4}$ Idem. 
45 Après l'indépendance du Congo en 1960, elle prend le nom Revue juridique d'Afrique centrale, pour être rebaptisée Revue Juridique du Congo en 1964 et Revue juridique du Zaïre en 1967.

46 Société d'Études juridiques. Fête du premier anniversaire...,op. cit.,p. 51.

47 Décret du 23 octobre 1926, dans Bulletin officiel du Congo belge, 1926, p. 448 et suiv.

48 À ce sujet, lire notamment les travaux de Jacques Vanderlinden ainsi que A. LAURO, "Une ceuvre d'étaiement et de reconstruction ». Notes sur la fabrique du droit coutumier, le pouvoir colonial et l'ordre du mariage dans le Congo Belge de l'entre-deux-guerres dans op. cit., éd. C. Braillon, L. Montel, B. Piret et P.-L. Plasman, p. 165-188.

$\underline{49}$ L'expression «tribunal indigène » (et sa variante «tribunal coutumier ») est largement consacrée par la littérature coloniale. Depuis les travaux de Martin Chanock, il apparait toutefois clairement que ces institutions sont très éloignées des juridictions existant avant leur réorganisation par les autorités coloniales. Aussi, nous plaçons cette expression entre guillemets afin de mettre en évidence la distance que nous prenons quant au caractère supposément authentique de cette institution.

50 Chronique : le départ de monsieur le procureur général Sohier, dans Revue de droit et de jurisprudence du Katanga, $\mathrm{n}^{0}$ 2, 15 février 1932, p. 67.

51 Éditorial..., op. cit., p. 1.

52 É. LAMY, op. cit., col. 393.

53 A. SOHIER, Fondons des cercles d'études juridiques, dans Journal des tribunaux d'Outre-Mer, $\mathrm{n}^{0} 37,15$ juillet 1953, p. 123.

$\underline{54}$ É. LAMY, op. cit., col. 394-395.

55 É. LAMY, op. cit., col. 392 et A. SOHIER, La Cour de cassation et la magistrature coloniale, dans Journal des tribunaux d'Outre-Mer, $\mathrm{n}^{0}$ 48, 15 juin 1954, p. 81-82.

56 Actuellement, quarante-quatre références bibliographiques d'Antoine Sohier sont reprises dans le répertoire bibliographique dédié à l'histoire du droit et de la justice. http://www.justhis.be/eprints/view/creators/Sohier=3AAntoine=3ANULL=3ANULL.html [consulté le 14 novembre 2017 via le site www.digithemis.be].

57 É. LAMY, op. cit., col. 393.

58 La bibliographie complète d'Antoine Sohier est parue dans le Bulletin des séances de l'ARSOM, Bruxelles, 1965, fasc. $\mathrm{n}^{0} 1$, p. 165-183.

59 Ces exemples se retrouvent notamment dans A. SOHIER, Les rapports entre les magistratures coloniale et métropolitaine..., op. cit.

60 P.-O. DE BRoux et B. PIRET, Légiférer au Congo belge. Le Conseil colonial, l'antichambre législative du pouvoir colonial, à paraitre.

$61 \mathrm{Au}$ sujet des étrangers présents dans l'ÉIC, lire les travaux de Lilian Nielsen consacrés aux 
Prosopographie et biographie : regards croisés sur la magistrature coloniale ...

Danois et de Rosario Giordano concernant les Italiens (R. GIORDANO, Belges et Italiens du CongoKinshasa. Récits de vie avant et après l'indépendance, Paris, Karthala, 2008).

62 Rapport du secrétaire d'État, E. Van Eetvelde, au Roi-Souverain, 21 mai 1897, dans Bulletin officiel de l’ÉIC, mai 1897, p. 192-193.

63 Rapport annuel sur l'administration du Congo belge, Bruxelles, Chambre des Représentants, 1909, 1911, 1913. Rapport annuel sur l'administration du Congo belge. Rapport sur l'activité de la colonie pendant la guerre, Bruxelles, Chambre des Représentants, 1919.

64 SPF AE, AA, fonds Service du Personnel d'Afrique, registre 94, fiches 243 et 355 ; registre 95, fiche 504.

65 Cet exemple met en exergue l'absence d'incitants au départ offerts pour la magistrature belge. A. SOHIER, Les rapports entre les magistratures coloniale et métropolitaine. Discours prononcé à la Conférence du Jeune Barreau de Bruxelles, Bruxelles, s. 1., 1926, p. 13.

66 Décret du Roi Souverain du 21 avril 1886 concernant l'organisation judiciaire, dans Bulletin officiel de l'ÉIC, 1896, p. 104 et suiv.

67 Peu de mémoires ont été conservés. Seule une cinquantaine d'entre eux, défendus dans les années 1930, peuvent être consultés dans le fonds Justice déposé au SPF AE.

68 É. LAMY, La magistrature coloniale belge dans Magistrats au temps des colonies, éd. A. ALLOTT, J.-P. ROYER, É. LAMY et al., Lille, Distique, 1988, p. 37.

69 L'École coloniale ouvre ses portes en 1903 et la section juridique accueille ses premiers étudiants en 1911. L'Université coloniale voit quant à elle le jour en 1920. Le droit colonial ne sera pas dispensé dans les universités belges avant les années 1920. C. VAN LEEUW, L'administration territoriale au Congo belge et au Ruanda Urundi. Fondements institutionnels et expérience vécue 1912-1960, Louvain-la-Neuve, Université catholique de Louvain, mémoire de licence, 1981, p. 143.

70 Les auteures de cette contribution remercient Amandine Dumont pour ces précieuses informations relatives à la formation des magistrats coloniaux, issues de la thèse qu'elle prépare actuellement sur le sujet.

71 A. GoHr, Organisation judiciaire de Congo belge, dans Les Novelles. Corpus Juris Belgici. Droit colonial, t. I, Bruxelles, Picard, 1931, p. 107.

72 L. MONTEL, op. cit., p. 66.

73 J. JADOT, Smets Charles, dans Biographie coloniale belge, t. V, Bruxelles, Institut Royal Colonial Belge, 1958, col.764-765.

74 Ce principe, institué par le décret du décret du 21 avril 1896, est ensuite consacré par les articles 18 et 18bis de la Charte coloniale. À ce sujet, lire notamment : A. GoHR, op. cit., p. 111-113. Cette situation n'est pas propre au Congo. Les magistrats coloniaux français sont également amovibles, lire notamment J.-C. FARCY, op. cit., p. 22.

75 En outre, Antoine Sohier considère que la pension coloniale ne permet pas de subvenir aux 
besoins d'un magistrat et de sa famille. A. SOHIER, L'organisation de la magistrature congolaise, dans Revue de Droit et de Jurisprudence du Katanga, $2^{\mathrm{e}}$ année, $\mathrm{n}^{0}$ 2, 15 février 1932, p. 45. À ce sujet, lire notamment K. LEYSEN, «Coloniser, c'est transporter! » Et alors la question de la main d'œuvre? De Belgische koloniale politiek met betrekking tot Belgisch Congo (1919-1929), Katholieke Universiteit Leuven, mémoire de licence, 2005-2006, p. 59-61 et 151-163.

76 A. SOHIER, Les rapports entre les magistratures coloniale et métropolitaine..., op. cit., p. 17-18.

77 Les archives coloniales sont actuelemment en cours de transfert vers les Archives générales du royaume. Il est à espérer que ce déménagement donne lieu à un renouvellement (voire à la rédaction) des instruments de recherche ad hoc. http://arch.arch.be/ index.php? $=$ =fr\&m =actualites\&r=toutes-les-actualites\&a $=2017-06-09$-transfert-des-archives-duspf-affaires-etrangeres [consulté le 15/11/2017].

PDF généré automatiquement le 2020-07-07 08:06:59

Url de l'article : https://popups.uliege.be:443/1370-2262/index.php?id=356 